EPJ Web of Conferences 27, 00006 (2012)

DOI: $10.1051 /$ epjconf/20122700006

(C) Owned by the authors, published by EDP Sciences, 2012

\title{
Covariance Matrix of a Double-Differential Doppler-Broadened Elastic Scattering Cross Section
}

\author{
G. Arbanas ${ }^{1, a}$, B. Becker ${ }^{2}$, R. Dagan ${ }^{3}$, M.E. Dunn ${ }^{1}$, N.M. Larson ${ }^{1}$, L.C. Leal ${ }^{1}$, and M.L. Williams ${ }^{1}$
}

1 Oak Ridge National Laboratory, P.O. Box 2008, Oak Ridge, TN 37831-6171, USA

2 Department of Mechanical, Aerospace and Nuclear Engineering, Rensselaer Polytechnic Institute, NES Bldg. 1-9, 3021 Tibbits Ave., Troy, NY 12180, USA

3 Institut für Neutronenphysik und Reaktortechnik, Forschungszentrum Karlsruhe Gmbh, Postfach 3640, 76021 Karlsruhe, Germany

\begin{abstract}
Legendre moments of a double-differential Doppler-broadened elastic neutron scattering cross section on ${ }^{238} \mathrm{U}$ are computed near the $6.67 \mathrm{eV}$ resonance at temperature $T=10^{3} \mathrm{~K}$ up to angular order 14 . A covariance matrix of these Legendre moments is computed as a functional of the covariance matrix of the elastic scattering cross section. A variance of double-differential Doppler-broadened elastic scattering cross section is computed from the covariance of Legendre moments.
\end{abstract}

\section{Introduction}

A general expression for temperature-dependent Legendre moments of a double-differential Doppler-broadened elastic scattering cross section was derived by Ouisloumen and Sanchez in [1]. However, its practical applications were limited to computations of the zeroth-order Legendre moment because higher order Legendre moments entail a timeconsuming computation of a triple-nested integral. A recursive algorithm that transforms this triple-nested integral into a single one via iterative application of the integrationby-parts method was designed and implemented in [2]. This algorithm enables accurate computation of Legendre moments of an arbitrary order in a way that bypasses the tedious programming of their explicit analytical expressions. We use this algorithm to compute the first fifteen Legendre moments and their covariance matrix.

We comment on convergence of a Legendre expansion of Doppler-broadened double-differential elastic neutron scattering cross section near a low energy resonance of ${ }^{238} \mathrm{U}$. A complementary stochastic treatment of thermal effects in [3] and [4] was used to validate a doubledifferential cross section computed via Legendre moment expansion. We also compute a covariance matrix of Legendre moments and use it to compute a variance of a doubledifferential Doppler-broadened elastic scattering cross section. In Section 2 essential formulae are listed, and in Section 3 numerical results are presented.

a e-mail: arbanasg@ornl.gov

Notice: This manuscript has been authored by UT-Battelle, LLC, under contract DE-AC05-00OR22725 with the U.S. Department of Energy. The United States Government retains and the publisher, by accepting the article for publication, acknowledges that the United States Government retains a non-exclusive, paid-up, irrevocable, world-wide license to publish or reproduce the published form of this manuscript, or allow others to do so, for United States Government purposes.

\section{Formalism}

A double-differential elastic scattering cross section in the laboratory frame at temperature $T$ can be expanded in Legendre polynomials as

$$
\sigma_{s}^{T}\left(E \rightarrow E^{\prime}, \mu_{\mathrm{lab}}\right)=\sum_{n \geq 0}\left(n+\frac{1}{2}\right) \sigma_{s n}^{T}\left(E \rightarrow E^{\prime}\right) P_{n}\left(\mu_{\mathrm{lab}}\right),
$$

where $\mu_{\text {lab }} \equiv \cos \angle\left(\mathbf{v}, \mathbf{v}^{\prime}\right), \mathbf{v}$ and $\mathbf{v}^{\prime}$ are the initial and the final velocity corresponding to the initial and the final energy $E$ and $E^{\prime}$ in the laboratory frame, respectively, and $P_{n}\left(\mu_{\text {lab }}\right)$ are the Legendre polynomials. It is noted that the angular distribution in the lab frame, Eq. (1), is anisotropic for $n \geq 1$ even when it is isotropic in the center-of-mass frame.

Low-energy elastic neutron scattering in the center-ofmass frame can be approximated to a high accuracy by isotropic $s$-wave scattering. An expression for the $n^{\text {th }}$-order Legendre moment (in the lab frame) of an isotropic angular distribution in the center-of-mass frame taken from [1] is:

$$
\sigma_{s n}^{T}\left(E \rightarrow E^{\prime}\right)=\frac{\beta^{5 / 2}}{4 E} e^{E / k T} \int_{0}^{\infty} t \sigma_{s}^{\mathrm{tab}}\left(E^{\prime \prime}(t)\right) e^{-t^{2} / A} \psi_{n}(t) \mathrm{d} t,
$$

where $E$ and $E^{\prime}$ are the incident and outgoing neutron energies in the laboratory frame, $T$ is the temperature in degrees Kelvin, $k$ is the Boltzmann constant, $\beta \equiv(A+1) / A$, $A$ is the target mass in units of neutron mass $m, E^{\prime \prime}(t) \equiv$ $\beta k T t^{2} / A$ is an energy in the lab frame, and $\sigma_{s}^{\text {tab }}\left(E_{\mathrm{lab}}\right)$ is a tabulated elastic scattering cross section ${ }^{1}$ at zero degrees Kelvin. The $\psi_{n}(t)$ is computed by Eqs. (13-26) of [2], where $\psi_{n}(t)$ is the $\psi_{n 0}(t)$ in a generalized notation introduced in [2]. The integration variable $t$ is dimensionless.

Legendre moments in Eq. (2) can be written compactly as functionals of $\sigma_{s}^{\mathrm{tab}}$ :

$$
\sigma_{s n}^{T}\left(E \rightarrow E^{\prime}\right)=\hat{O}_{n}^{T}\left(E \rightarrow E^{\prime}\right)\left[\sigma_{s}^{\mathrm{tab}}\right]
$$

1 Usually given as a function of energy in the lab frame. 
where the operator $\hat{O}_{n}^{T}\left(E \rightarrow E^{\prime}\right)$ is defined by equivalence of Eq. (2) and Eq. (3). The temperature, the incoming and the outgoing energy can be combined into a vector variable $\mathbf{x} \equiv\left(T, E, E^{\prime}\right)$, so that Eq. (3) becomes

$$
\sigma_{s n}(\mathbf{x})=\hat{O}_{n}(\mathbf{x})\left[\sigma_{s}^{\mathrm{tab}}\right]
$$

A covariance matrix of Legendre moments is then

$$
M_{n n^{\prime}}(\mathbf{x}, \mathbf{y}) \equiv\left\langle\delta \sigma_{s n}(\mathbf{x}) \delta \sigma_{s n^{\prime}}(\mathbf{y})\right\rangle=\hat{O}_{n}(\mathbf{x}) \hat{O}_{n^{\prime}}(\mathbf{y})\left[V_{s}\right],
$$

where

$$
V_{s}\left(E, E^{\prime}\right) \equiv\left\langle\delta \sigma_{s}^{\mathrm{tab}}(E) \delta \sigma_{s}^{\mathrm{tab}}\left(E^{\prime}\right)\right\rangle
$$

is a covariance matrix of a zero-temperature elastic scattering cross section ${ }^{2}$. A double nested integral implicit in Eq. (5) is symmetric because the covariance matrix $V$ in the integrand is symmetric. Combining Eqs. (1) and (5) yields a covariance matrix of a double-differential cross section:

$$
\begin{array}{r}
\left\langle\delta \sigma_{s}\left(\mathbf{x}, \mu_{\mathrm{lab}}\right) \delta \sigma_{s}\left(\mathbf{y}, \mu_{\mathrm{lab}}^{\prime}\right)\right\rangle=\sum_{n, n^{\prime}}\left(n+\frac{1}{2}\right)\left(n^{\prime}+\frac{1}{2}\right) \\
\times P_{n}\left(\mu_{\mathrm{lab}}\right) P_{n^{\prime}}\left(\mu_{\mathrm{lab}}^{\prime}\right) M_{n n^{\prime}}(\mathbf{x}, \mathbf{y})
\end{array}
$$

We set $\mathbf{y}=\mathbf{x}$ to compute a covariance matrix of Legendre moments, $M_{n n^{\prime}}(\mathbf{x}, \mathbf{x})$, and then we set $\mu_{\mathrm{lab}}^{\prime}=\mu_{\mathrm{lab}}$ to compute a variance of double-differential elastic scattering cross section, $\left\langle\left(\delta \sigma_{s}\left(\mathbf{x}, \mu_{1 \mathrm{ab}}\right)\right)^{2}\right\rangle$. Assuming that $T, E$, and $E^{\prime}$ are known precisely, then the covariance matrix of Legendre moments in Eq. (5) and a covariance matrix of a double-differential scattering in Eq. (7) are completely determined by the elastic scattering covariance matrix $V\left(E, E^{\prime}\right)$.

\section{Results}

Computations were performed for incoming neutron energies just below and just above the ${ }^{238} \mathrm{U} 6.67 \mathrm{eV}$ resonance, namely for $E=6.5$ and $6.8 \mathrm{eV}$, and for temperature $T=10^{3} \mathrm{~K}$. The ${ }^{238} \mathrm{U}$ elastic neutron scattering cross section $\sigma_{s}^{\mathrm{tab}}(E)$ at $0 \mathrm{~K}$ used in Eq. (2) is based on ENDF/B-VII Release 0 and is plotted in Fig. 1. The fifteen Legendre moments computed via Eq. (2) are plotted in Figs. 2 and 3 for incoming energies $E=6.5 \mathrm{eV}$ and $E=6.8 \mathrm{eV}$, respectively. A covariance matrix $V_{s}\left(E, E^{\prime}\right)$ of ${ }^{238} \mathrm{U}$ total elastic neutron scattering cross section at $T=0 \mathrm{~K}$ that was used to compute the covariance in Eq. (5) is plotted in Fig. 4. The peak in the covariance matrix corresponds to the 6.67 $\mathrm{eV}$ resonance.

For an incoming energy $E=6.5 \mathrm{eV}$ that is just below the resonance energy, the thermal motion of ${ }^{238} \mathrm{U}$ causes the average outgoing neutron energy to be greater than the incoming energy ("up-scattering"), $\left\langle E^{\prime}\right\rangle=6.60 \mathrm{eV}$ is greater than $E=6.5 \mathrm{eV}$, as can be seen in Fig. 5. For an incoming energy $E=6.8 \mathrm{eV}$, which is just above the resonance energy, the effect is in reverse ("down-scattering"), $\left\langle E^{\prime}\right\rangle=6.67 \mathrm{eV}$ is less than $E=6.8 \mathrm{eV}$, as can be seen in Fig. 7.

For a given incoming energy $E$, the integral in Eq. (2) was computed on a $t$-mesh that corresponds ${ }^{3}$ to the energy range $[E-0.6, E+0.6] \mathrm{eV}$, using a dimensionless mesh size

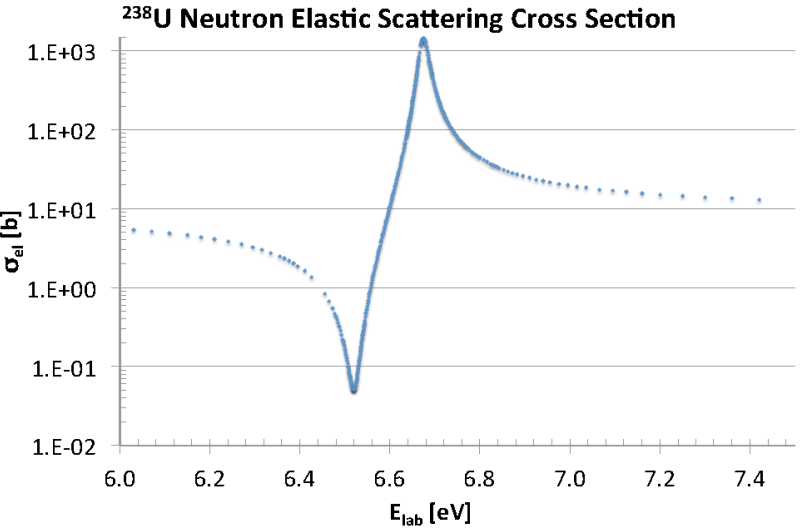

Fig. 1. ${ }^{238} \mathrm{U}$ total elastic neutron scattering cross section $\sigma_{s}^{\mathrm{tab}}(E)$ at $0 \mathrm{~K}$ based on ENDF/B-VII Release 0 was used in Eq. (2).

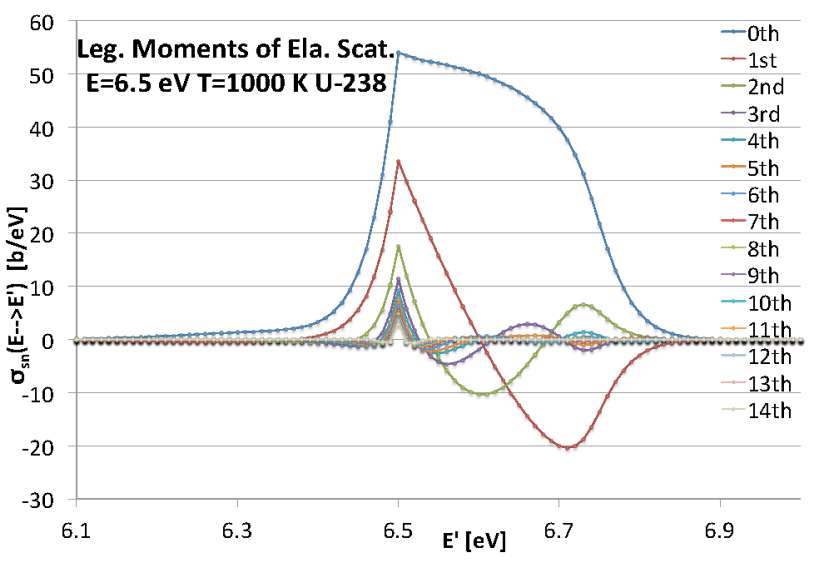

Fig. 2. Legendre moments $\sigma_{n}^{T}\left(E \rightarrow E^{\prime}\right), n=0,1, \ldots, 14$ of ${ }^{238} \mathrm{U}$ elastic neutron scattering cross section at incoming energy $E=$ $6.5 \mathrm{eV}$ and temperature $T=10^{3} \mathrm{~K}$ were computed via Eq. (2) and used in Eq. (1) to produce a double-differential elastic scattering cross section in Fig. 5. Legendre moments of a corresponding scattering kernel are $P_{s n}^{T}\left(E \rightarrow E^{\prime}\right)=\sigma_{s n}^{T}\left(E \rightarrow E^{\prime}\right) / \sigma_{s}^{T}(E)$, where $\sigma_{s}^{T}(E)=\int_{0}^{\infty} \sigma_{s 0}^{T}\left(E \rightarrow E^{\prime}\right) \mathrm{d} E^{\prime}=14.3 \mathrm{~b}$.

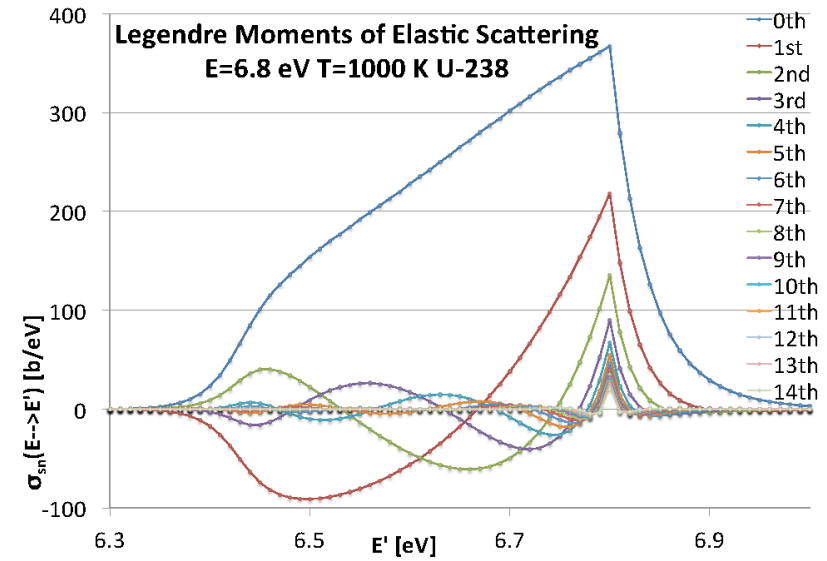

Fig. 3. As in Fig. 2 but for incoming neutron energy $E=6.8 \mathrm{eV}$; since $E$ is above the $6.67 \mathrm{eV}$ resonance energy, down-scattering probability is dominating. $\sigma_{s}^{T}(E)=103.4 \mathrm{~b}$.

\footnotetext{
${ }^{2}$ Energies $E$ and $E^{\prime}$ in $V\left(E, E^{\prime}\right)$ are generic variable names.

3 Via $E(t) \equiv \beta k T t^{2} / A$.
} 


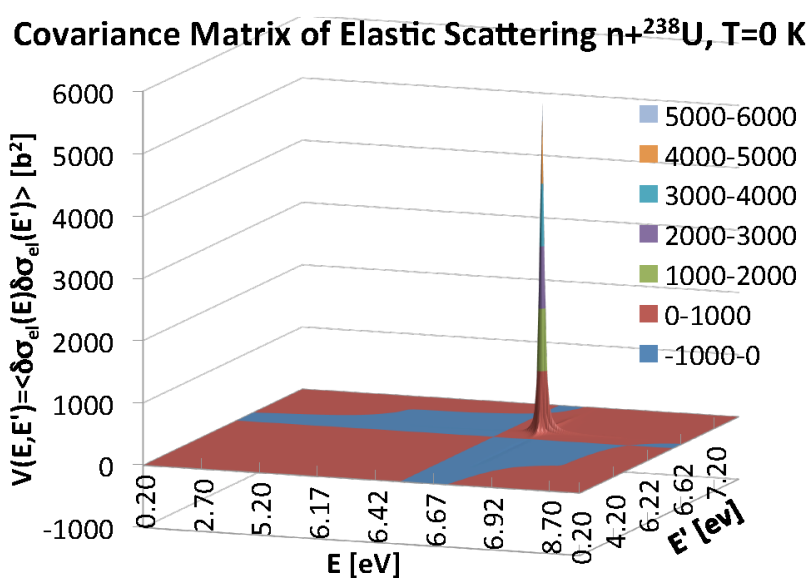

Fig. 4. Covariance matrix $V_{s}\left(E, E^{\prime}\right)$ of ${ }^{238} \mathrm{U}$ total elastic neutron scattering cross section at $T=0 \mathrm{~K}$ which was used in Eq. (5).

$\Delta t=0.01$. The outgoing energies $E^{\prime}$ are given on a mesh spanning $[E-0.5, E+0.5] \mathrm{eV}$ in increments of $\Delta E^{\prime}=0.01$ $\mathrm{eV}$, while the angular mesh is from 0 to $180^{\circ}$ in increments of $10^{\circ}$; a combined two-dimensional $\left(E^{\prime}, \theta\right)$ mesh was used in Figs. 5-8.

The fifteen Legendre moments, plotted in Figs. 2 and 3 , are sufficiently many to attain a convergence of the Legendre expansion in Eq. (1) everywhere except for $E^{\prime}=E$ and at backward angles. This slow convergence may be understood by observing that: 1) at $E^{\prime}=E$ Legendre moments have maxima that decrease relatively slowly with increasing order ${ }^{4}$ so that the factor $\left(n+\frac{1}{2}\right)$ appearing in the expansion softens the decrease of their product, and 2) for $\mu_{\text {lab }}=-1$ Legendre moments change sign as $P_{n}(-1)=$ $(-1)^{n}$. A combined effect of this is that the (short) spike that is seen in Figs. 5 and 7 at $E=E^{\prime}$ and $\mu_{\text {lab }}=-1$ decreases slowly magnitude-wise and changes its sign for each term in Eq. (1). In view of this slow convergence, a direct computation of the double-differential elastic scattering that was expressed as a doubly nested integral in [5] may be an alternative to be considered. Nevertheless, the up- and down-scattering caused by the $6.67 \mathrm{eV}^{238} \mathrm{U}$ resonance is clearly visible in Figs. 5 and 7 , respectively. Small negative values appear in the double-differential cross section because a finite number of Legendre moments is used. The absolute uncertainty in the double-differential elastic cross section is plotted in Figs. 6 and 8 for incoming energies $E=6.5 \mathrm{eV}$ and $E=6.8 \mathrm{eV}$, respectively. A relative uncertainty corresponding to this absolute uncertainty is nearly constant and equal to the relative uncertainty of the elastic scattering cross section at the peak of the $6.67 \mathrm{eV}$ resonance, approximately $5 \%$. This is an indication that the peak in the covariance matrix corresponding to the $6.67 \mathrm{eV}$ resonance in Fig. 4 makes a dominant contribution to the integral in Eqs. (5) and (7).
Double-Differential Elastic Scattering Cross Section

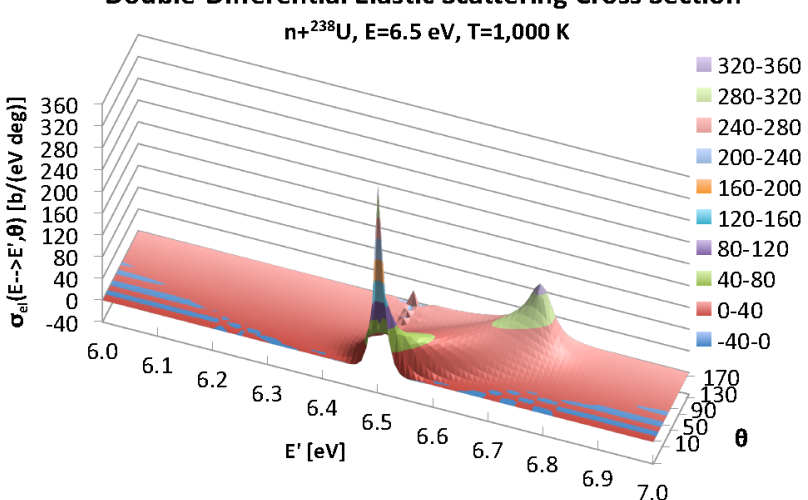

Fig. 5. $\sigma_{s}^{T}\left(E \rightarrow E^{\prime}, \mu_{\mathrm{lab}}\right)$ computed via Eq. (1) for $E=6.5 \mathrm{eV}$ and $T=10^{3} \mathrm{~K}$, where $\mu_{\mathrm{lab}}=\cos (\theta)$, using the first fifteen Legendre moments shows good convergence everywhere except for $E^{\prime}=E$ at backward angles where many Legendre moments are needed to improve convergence. The up-scattering due to the $6.67 \mathrm{eV}$ resonance is clearly visible.

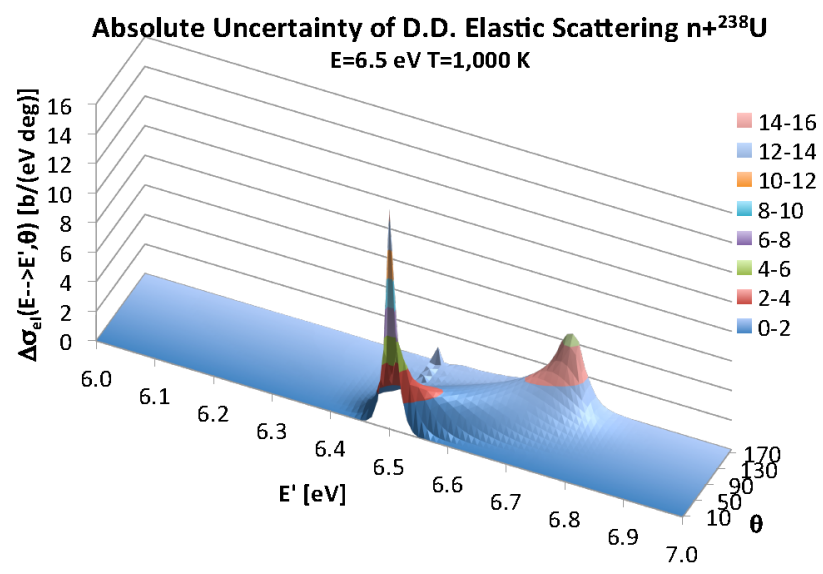

Fig. 6. A plot of uncertainty of $\sigma_{s}^{T}\left(E \rightarrow E^{\prime}, \mu_{\mathrm{lab}}\right)$ from Fig. 5 . The uncertainty is computed via Eq. (7) using the first fifteen Legendre moments. Convergence is satisfactory except for $E^{\prime}=$ $E$ at backward angles, just as in Fig. 5. A dominant contribution to the uncertainty comes from a large peak in the covariance at the $6.67 \mathrm{eV}^{238} \mathrm{U}$ resonance seen in Fig. 4.

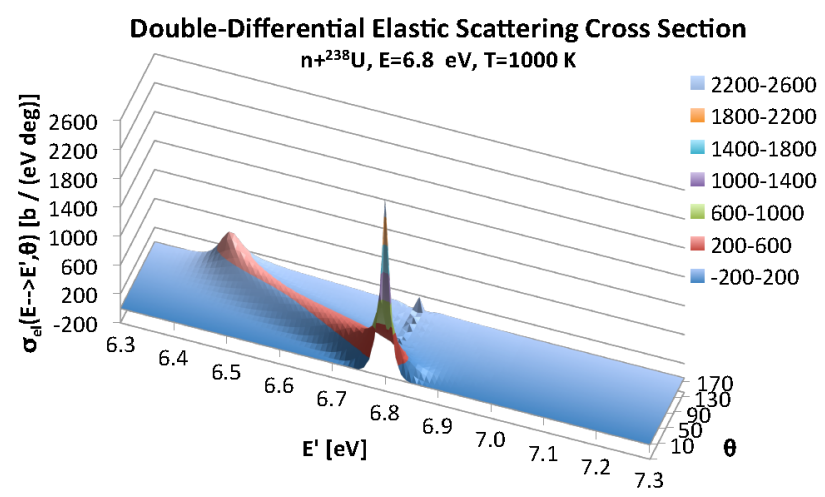

Fig. 7. As in Fig. 5 but for incoming energy $E=6.8 \mathrm{eV}$; since $E$ is above the $6.67 \mathrm{eV}$ resonance energy down-scattering probability is dominating. 
EPJ Web of Conferences

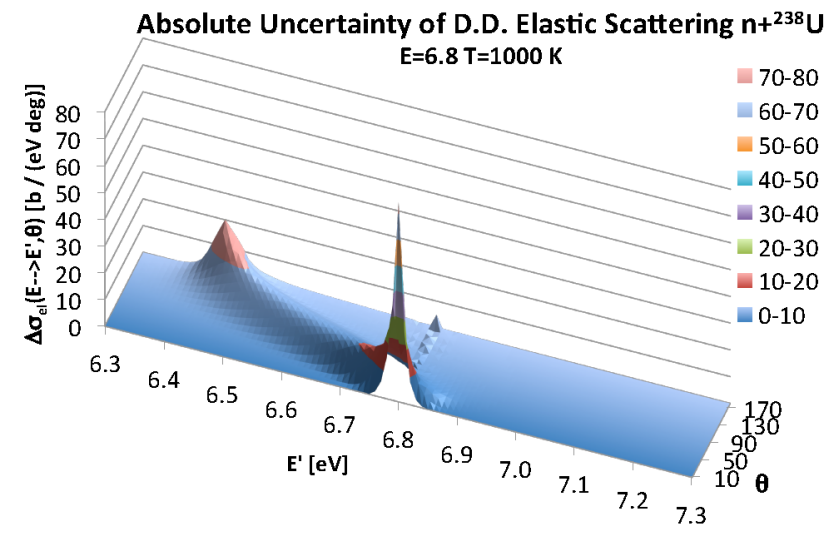

Fig. 8. As in Fig. 6 but for incoming energy $E=6.8 \mathrm{eV}$.

This manuscript has been authored by UT-Battelle, LLC, under contract DE-AC05-00OR22725 with the U.S. Department of Energy. The authors would like to thank Dr. Marco Pigni for suggesting the functional notation in Eq. (3), Dr. Dorothea (Doro) Wiarda for help with programming, and Dr. Sedat Goluoglu for creating the zero temperature elastic scattering cross section file.

\section{References}

1. M. Ouisloumen, R. Sanchez, Nucl. Sci. Eng. 107, (1991) 189

2. G. Arbanas, M.E. Dunn, N.M. Larson, L.C. Leal, M.L. Williams, B. Becker, R. Dagan, Proc. International Conference on Mathematics and Computational Methods Applied to Nuclear Science and Engineering (MC 2011), May 8-12, 2011, Rio de Janeiro, Brazil (LAS/American Nuclear Society 2011)

3. B. Becker, R. Dagan, G. Lohnert, Ann. Nucl. Energy 36, (2009) 470

4. B. Becker, On the Influence of the Resonance Scattering Treatment in Monte Carlo Codes on High Temperature Reactor Characteristics (Doctor of Engineering Thesis, Universität Stuttgart, Stuttgart, Germany 2010)

5. W. Rothenstein, Ann. Nucl. Energy 31, (2004) 9 